\title{
Verification of the OWL-Time Ontology
}

\author{
Michael Grüninger \\ Department of Mechanical and Industrial Engineering, \\ University of Toronto, Toronto, Ontario, Canada M5S 3G8
}

\begin{abstract}
Ontology verification is concerned with the relationship between the intended structures for an ontology and the models of the axiomatization of the ontology. The verification of a particular ontology requires characterization of the models of the ontology up to isomorphism and a proof that these models are equivalent to the intended structures for the ontology. In this paper we provide the verification of the ontology of time introduced by Hobbs and Pan, which is a first-order axiomatization of OWL-Time. We identify five modules within this ontology and present a complete account of the metatheoretic relationships among the modules and between other time ontologies for points and intervals.
\end{abstract}

\section{Introduction}

Over the years, a number of first-order ontologies for time have been proposed. In addition to ontologies for timepoints and ontologies for time intervals ([2]), there are also ontologies that axiomatize both timepoints and time intervals together with the relationships between them. More recently, Hobbs and Pan ([10], [12]) have proposed a first-order axiomatization $T_{\text {owltime }}$ of OWL-Time 1 as an ontology of time for the Semantic Web that also includes both timepoints (referred to as instants) and intervals.

The primary objective of this paper is to provide a characterization of the models of $T_{\text {owltime }}$ up to isomorphism using the notion of theory reducibility from [6]. This will lead to a modularization of $T_{\text {owltime }}$ and allow us to identify incorrect or missing axioms in the current axiomatization of $T_{\text {owltime }}$. Finally, we will also use this reduction to compare $T_{\text {owltime }}$ to other time ontologies for points and intervals, and address the question as to whether $T_{\text {owltime }}$ forms an adequate core theory for time ontologies, or whether it is too weak or too strong to play such a role.

\section{Ontology Verification}

Our methodology revolves around the application of model-theoretic notions to the design and analysis of ontologies. The semantics of the ontology's terminology can be characterized by a set of structures, which we refer to as the set of intended structures for the ontology. Intended structures are specified with respect to the models of wellunderstood mathematical theories (such as partial orderings, lattices, incidence structures, geometries, and algebra). The extensions of the relations in an intended structure are then specified with respect to properties of these models.

1 http: / / www .w3 . org/TR/owl-time/ 
Why do we care about ontology verification? The relationship between the intended models and the models of the axiomatization plays a key role in the application of ontologies in areas such as semantic integration and decision support. Software systems are semantically integrated if their sets of intended models are equivalent. In the area of decision support, the verification of an ontology allows us to make the claim that any inferences drawn by a reasoning engine using the ontology are actually entailed by the ontology's intended models. If an ontology's axiomatization has unintended models, then it is possible to find sentences that are entailed by the intended models, but which are not provable from the axioms of the ontology. The existence of unintended models also prevents the entailment of sentences or a possible barriers to interoperability.

With ontology verification, we want to characterize the models of an ontology up to isomorphism and determine whether or not these models are elementarily equivalent to the intended structures of the ontology. From a mathematical perspective this is formalized by the notion of representation theorems. The primary challenge for someone attempting to prove representation theorems is to characterize the models of an ontology up to isomorphism. For this we use the following notion from [6]:

Definition 1. A class of structures $\mathfrak{M}$ can be represented by a class of structures $\mathfrak{N}$ iff there is a bijection $\varphi: \mathfrak{M} \rightarrow \mathfrak{N}$ such that for any $\mathcal{M} \in \mathfrak{M}, \mathcal{M}$ is definable in $\varphi(\mathcal{M})$ and $\varphi(\mathcal{M})$ is definable in $\mathcal{M}$.

The key to ontology verification is that a theorem about the relationship between the class of the ontology's models and the class of intended structures can be replaced by a theorem about the relationship between the ontology (a theory) and the theory axiomatizing the intended structures (assuming that such axiomatization is known). We can use automated reasoners to prove this relationship and thus verify an ontology in a (semi-)automated way.

The relationship between theories $T_{A}$ and $T_{B}$ is the notion of interpretation ([5]), which is a mapping from the language of $T_{A}$ to the language of $T_{B}$ that preserves the theorems of $T_{A}$. If there is an interpretation of $T_{A}$ in $T_{B}$, then there exists a set of sentences (referred to as translation definitions) in the language $L_{A} \cup L_{B}$ of the form

$$
(\forall \bar{x}) p_{i}(\bar{x}) \equiv \varphi(\bar{x})
$$

where $p_{i}(\bar{x})$ is a relation symbol in $L_{A}$ and $\varphi(\bar{x})$ is a formula in $L_{B}$. Translation definitions will be used extensively in the proofs of theorems later in the paper.

We will say that two theories $T_{A}$ and $T_{B}$ are definably equivalent iff they are mutually interpretable, i.e. $T_{A}$ is interpretable in $T_{B}$ and $T_{B}$ is interpretable in $T_{A}$.

The key to using theorem proving and model finding to support ontology verification is the following theorem $([\overline{6}])$ :

Theorem 1. A theory $T_{1}$ is definably equivalent with a theory $T_{2}$ iff the class of models $\operatorname{Mod}\left(T_{1}\right)$ can be represented by $\operatorname{Mod}\left(T_{2}\right)$.

Let $\mathfrak{M}^{\text {intended }}$ be the class of intended structures for the ontology, and let $T_{\text {onto }}$ be the axiomatization of the ontology. The necessary direction of a representation theorem 
(i.e. if a structure is intended, then it is a model of the ontology's axiomatization) can be stated as

$$
\mathcal{M} \in \mathfrak{M}^{\text {intended }} \Rightarrow \mathcal{M} \in \operatorname{Mod}\left(T_{\text {onto }}\right)
$$

If we suppose that the theory that axiomatizes $\mathfrak{M}^{\text {intended }}$ is the union of some previously known theories $T_{1}, \ldots, T_{n}$, then by Theorem 1 we need to show that $T_{\text {onto }}$ interprets $T_{1} \cup \ldots \cup T_{n}$. If $\Delta$ is the set of translation definitions for this interpretation, then the necessary direction of the representation theorem is equivalent to the following reasoning task:

$$
T_{\text {onto }} \cup \Delta \models T_{1} \cup \ldots \cup T_{n}
$$

The sufficient direction of a representation theorem (any model of the ontology's axiomatization is also an intended structure) can be stated as

$$
\mathcal{M} \in \operatorname{Mod}\left(T_{\text {onto }}\right) \Rightarrow \mathcal{M} \in \mathfrak{M}^{\text {intended }}
$$

In this case, we need to show that $T_{1} \cup \ldots \cup T_{n}$ interprets $T_{\text {onto }}$. If $\Pi$ is the set of translation definitions for this interpretation, the sufficient direction of the representation theorem is equivalent to the following reasoning task:

$$
T_{1} \cup \ldots \cup T_{n} \cup \Pi \models T_{\text {onto }}
$$

Proving these two entailment problems constitutes the reducibility theorem for $T_{\text {onto }}$; the set of theories $T_{1}, \ldots, T_{n}$ form the reduction of $T_{\text {onto }}$.

All of the theories introduced in this paper are being investigated in the context of the COLORE (Common Logic Ontology Repository) project, which is building an open repository of first-order ontologies that serve as a testbed for ontology evaluation and integration techniques, and that can support the design, evaluation, and application of ontologies in first-order logic. We identify the theories for the reduction by searching through the COLORE ontology repository for ontologies that are definably equivalent to the ontology that we are verifying, and then prove that the mappings between the ontologies are correct.

\section{Modularization of OWL-Time}

The verification of the OWL-Time Ontology $T_{\text {owltime }}$ will also provide a decomposition of the ontology into a set of subtheories which are related by conservative extension. We will not present the verification of the entire OWL-Time Ontology in this paper; rather we will focus on the subtheory $T_{\text {timespan }}$ of $T_{\text {owltime }}$ that omits the axioms for durations and dates. Furthermore, throughout the paper we will identify additional axioms that are required to prove the representation theorems, leading to the specification of a theory $T_{\text {timespan }}^{*}$ as an extension of $T_{\text {timespan }}$. By the end of the paper, we will show that $T_{\text {timespan }}^{*}$ is definably equivalent to the following theories (which will be introduced and discussed in detail in the following sections) from COLORE:

$$
T_{\text {linear_order }} \cup T_{\text {pseudo_complete }} \cup T_{\text {log }} \cup T_{\text {weak_planar }} \cup T_{\text {diamond }}
$$


In Figure 1 we can see which subsets of these theories interpret subtheories of $T_{\text {owltime }}$, and this set of subtheories 2 constitutes the modules within $T_{\text {owltime. }}$ In this way, modules within OWL-Time are obtained by identifying ontologies within the COLORE ontology repository that are definably equivalent to subtheories of OWL-Time. In the following sections, we will specify the reduction theorems for each of these subtheories, culminating in the representation theorem for $T_{\text {owltime }}^{*}$.

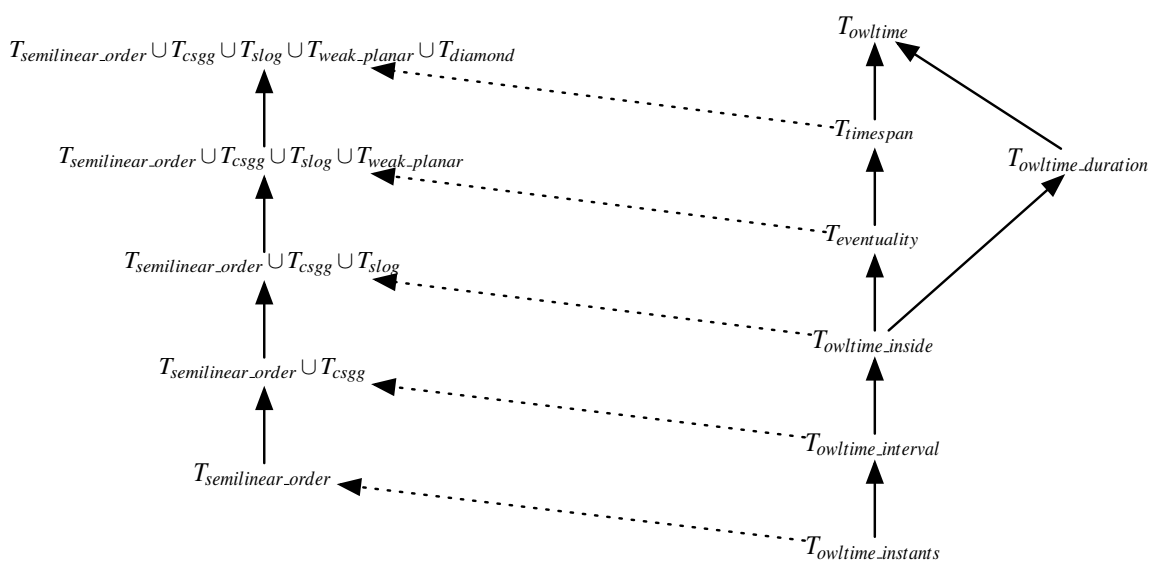

Fig. 1. Relationships between the subtheories of $T_{\text {owltime }}$ and the theories from COLORE which are used to prove the representation theorem for $T_{\text {owltime }}$. Solid lines denote conservative extension and dotted lines denote interpretability between theories.

\section{Instants and Intervals}

The first two modules within OWL-Time that we will consider are $T_{\text {owltime_instant }}$ and $T_{\text {owltime_interval }}$, which axiomatize fundamental intuitions about timepoints (Instants) and time intervals (Intervals). Besides the objective of ontology verification, we will also be interested in using the reduction of $T_{\text {owltime_interval }}$ to determine the relationship to other ontologies for timepoints and time intervals. Given an existing ontology $T$ of timepoints and intervals, we will identify the theory that is a common extension of both $T_{\text {owltime_interval }}$ and $T$, and also identify the common subtheory of Towltime_interval and $T$.

\footnotetext{
${ }^{2}$ The original first-order axiomatization of $T_{\text {owltime }}$ can be found at http://www.cs.rochester.edu/ ferguson/daml/ daml-time-nov2002.txt
}

The CLIF (Common Logic Interchange Format) axiomatization of the subtheories of $T_{\text {owltime }}$ discussed in this paper can be found at

http://stl.mie.utoronto.ca/colore/time/owltime_instants.clif http://stl.mie.utoronto.ca/colore/time/owltime_interval.clif http://stl.mie.utoronto.ca/colore/time/owltime_inside.clif http://stl.mie.utoronto.ca/colore/time/eventuality.clif http://stl.mie.utoronto.ca/colore/time/timespan.clif 


\subsection{Time Ontologies for Points}

Ontologies for time points have been studied in [2] and [9], and all axiomatize an ordering relation over the points. Although Hobbs and Pan rightly argue that a restriction to linear orders is too strong, allowing arbitrary partial orderings is arguably too weak. We consider the following extension:

Definition 2. $T_{\text {owltime_instant_s }}$ is the extension of $T_{\text {owltime_instant }}$ with the axiom $(\forall x, y) \operatorname{Instant}(x) \wedge \operatorname{Instant}(y) \supset(\exists z)($ before $(z, x) \vee(z=x)) \wedge($ before $(z, y) \vee(z=y)))$

It is straightforward to see that the resulting ordering on the set of instants is a semilinear ordering [4] as axiomatized by the theory $T_{\text {semilinear_orderin }}$. 3 .

\subsection{Time Ontologies for Points and Intervals}

In his Catalog of Temporal Theories [9], Hayes introduced three time ontologies that axiomatize both timepoints and time intervals. The endpoints theory combines the language of intervals and points by defining the functions beginof, endof, and between to relate intervals to points and vice-versa. This theory imports the axioms of linear_point that define the binary before relation between timepoints as transitive and irreflexive, and impose the condition that all timepoints are linearly ordered and infinite in both directions. The vector_continuum theory is a theory of timepoints and intervals that introduces the notion of orientation of intervals. It also imports theory linear_point. In this theory single-point intervals, known as moments, are defined as intervals whose beginof and endof points are the same. The point_continuum theory combines intervals and points by defining the relation in that relates a point to the interval it is contained in. The verification of these three time ontologies is given in [7].

The theory $\mathcal{I} \mathcal{Q}$ in [13] (which we will refer to as $T_{\text {vila }}$ in this paper) is a common subtheory for all of the ontologies of time points and intervals. Later in the paper, we will characterize the relationship between $T_{\text {vila }}$ and $T_{\text {owltime_interval }}$. To lay the groundwork for this characterization, we review the classes of structures that will be used for the reducibility theorem for these theories.

Graphical Incidence Structures. The basic building blocks for the models presented in this paper are based on the notion of incidence structures ([3]).

Definition 3. A k-partite incidence structure is a tuple $\mathbb{I}=\left(\Omega_{1}, \ldots, \Omega_{k}\right.$, in $)$, where $\Omega_{1}, \ldots, \Omega_{k}$ are sets with

$$
\Omega_{i} \cap \Omega_{j}=\emptyset, i \neq j
$$

and

$$
\text { in } \subseteq\left(\bigcup_{i \neq j} \Omega_{i} \times \Omega_{j}\right)
$$

Two elements of $\mathbb{I}$ that are related by in are called incident.

\footnotetext{
${ }^{3}$ http://stl.mie.utoronto.ca/colore/ordering/ semilinear_ordering.clif
} 
The models of the time ontologies in this paper will be constructed using special classes of incidence structures.

Definition 4. An strong graphical incidence structure is a bipartite incidence structure

$$
\mathbb{S}=\left\langle X, Y, \text { in }^{\mathbf{S}}\right\rangle
$$

such that all elements of $Y$ are incident with either one or two elements of $X$, and for each pair of points $\mathbf{p}, \mathbf{q} \in X$ there exists a unique element in $Y$ that is incident with both $\mathbf{p}$ and $\mathbf{q}$.

The class of strong graphical incidence structures is axiomatized by $T_{\text {strong_graphica }}$

Definition 5. A loop graphical incidence structure is a bipartite incidence structure

$$
\mathbb{S}=\left\langle X, Y, \mathbf{i n}^{\mathbf{S}}\right\rangle
$$

such that all elements of $Y$ are incident with either one or two elements of $X$, and for each pair of points $\mathbf{p}, \mathbf{q} \in X$ there exists a unique element in $Y$ that is incident with both $\mathbf{p}$ and $\mathbf{q}$, and for each point $\mathbf{r} \in X$ there exists a unique element in $Y$ that incident only with $\mathbf{r}$.

The class of loop graphical incidence structures is axiomatized by $T_{\text {loop_graphica }} 5$.

These classes of incidence structures get their names from graph-theoretic representation theorems of their own.

Definition 6. A graph $G=(V, E)$ consists of a nonempty set $V$ of vertices and a set $E$ of ordered pairs of vertices called edges.

An edge whose vertices coincide is called a loop. A graph with no loops or multiple edges is a simple graph.

A complete graph is a graph in which each pair of vertices is adjacent.

Theorem 2. Let $G=(V, E)$ be a complete graph.

A bipartite incidence structure is a strong graphical incidence structure iff it is isomorphic to $\mathbb{I}=(V, E, \in)$.

Theorem 3. Let $G=(V, E)$ be a complete graph with loops.

A bipartite incidence structure is a loop graphical incidence structure iff it is isomorphic to $\mathbb{I}=(V, E, \in)$.

These representation theorems show that there is a one-to-one correspondence between the particular class of incidence structures and the given class of graphs; in so doing, we have a characterization of the incidence structures up to isomorphism.

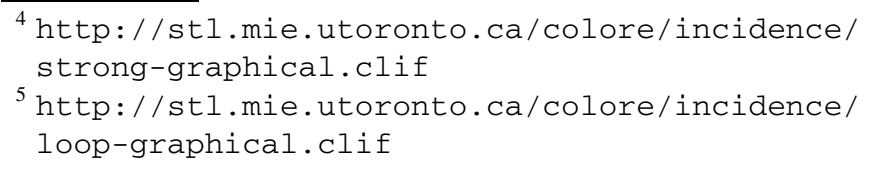


Reducibility Theorems for Points and Intervals. We will ultimately be interested in determining the relationship between the theory $T_{\text {vila }}$ and $T_{\text {owltime_interval }}$. On the one hand, one of the design objectives for OWL-Time was to be a relatively weak theory that could be consistently extended to other time ontologies. On the other hand, $T_{\text {vila }}$ is the common subtheory of existing time ontologies for points and intervals.

The approach taken in this paper is to compare different time ontologies by comparing the theories in their reductions. Using the axiomatizations of these classes of incidence structures, we can prove the following reducibility theorem for $T_{\text {vila }}$.

Theorem 4. $T_{\text {vila }}$ is definably equivalent to

$$
T_{\text {linear_order }} \cup T_{\text {strong_graphical }}
$$

Proof.

Using the same set of translation definitions, we can also prove the following:

Theorem 5. Let $T_{\text {moment }}$ be the extension of $T_{\text {vila }}$ with the axiom

$(\forall t)$ timepoint $(t) \supset(\exists i)$ timeinterval $(i) \wedge($ beginof $(i)=t) \wedge(\operatorname{endof}(i)=t)$

$T_{\text {moment }}$ is definably equivalent to

$$
T_{\text {linear_order }} \cup T_{\text {loop_graphical }}
$$

In the next section we will show how this theory $T_{\text {moment }}$ is definably equivalent to an extension of $T_{\text {owltime_interval }}$.

\subsection{Reducibility Theorems for Extensions of $\boldsymbol{T}_{\text {owltime_interval }}$}

Given that the time ontologies in [9] and [13] use linear orderings over timepoints, We introduce the following extensions and subtheories of $T_{\text {owltime_interval }}$ that impose a linear ordering on instants.

$T_{\text {owltime_linear }}$ is the extension of $T_{\text {owltime_interval }}$ with the axiom

$\left(\forall t_{1}, t_{2}\right) \operatorname{Instant}\left(t_{1}\right) \wedge \operatorname{Instant}\left(t_{2}\right) \supset\left(\right.$ before $\left(t_{1}, t_{2}\right) \vee$ before $\left.\left(t_{2}, t_{1}\right) \vee\left(t_{1}=t_{2}\right)\right)$

$T_{\text {owltime_e }}$ is the extension of $T_{\text {owltime_interval }}$ with the axiom

$$
(\forall i) \operatorname{Interval}(i) \supset\left(\exists t_{1}, t_{2}\right) \operatorname{begins}\left(t_{1}, i\right) \wedge \operatorname{ends}\left(t_{2}, i\right)
$$

$T_{\text {owltime_le }}$ is the extension of $T_{\text {owltime_linear }}$ with $T_{\text {owltime_e }}$.

$T_{\text {owltime_leu }}$ is the extension of $T_{\text {owltime_le }}$ with the axiom

$\left(\forall t_{1}, t_{2}, i_{1}, i_{2}\right) \operatorname{begins}\left(t_{1}, i_{1}\right) \wedge \operatorname{ends}\left(t_{2}, i_{1}\right) \wedge \operatorname{begins}\left(t_{1}, i_{2}\right) \wedge \operatorname{ends}\left(t_{2}, i_{2}\right) \supset\left(i_{1}=i_{2}\right)$

$T_{\text {owltime_m }}$ is the subtheory of $T_{\text {owltime }}$ without the axioms

$$
\begin{gathered}
(\forall t)(\operatorname{Instant}(t) \equiv \operatorname{begins}(t, t)) \\
(\forall t)(\operatorname{Instant}(t) \equiv \operatorname{ends}(t, t))
\end{gathered}
$$

The relationships between these theories are shown in Figure2. 


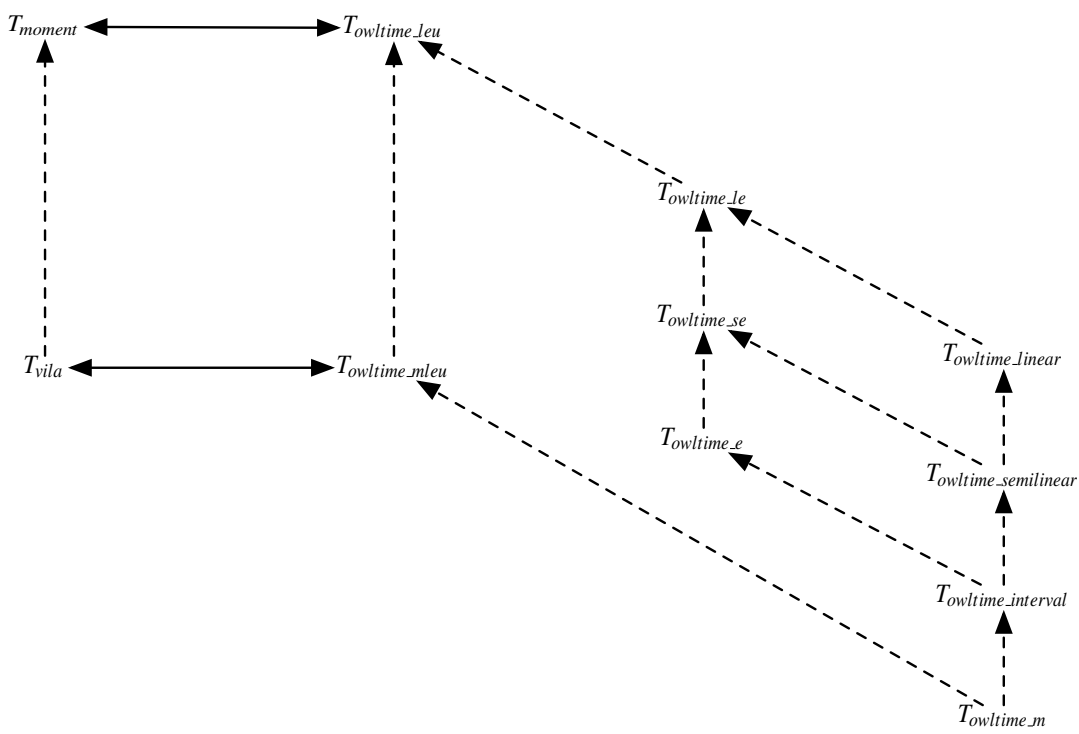

Fig. 2. Relationships between extensions of Towltime_interval and other time ontologies for points and intervals. Dotted lines denote nonconservative extension and solid lines denote definable equivalence.

Theorem 6. Towltime_leu is definably equivalent to

$$
T_{\text {linear_order }} \cup T_{\text {loop_graphical }}
$$

Proof. Let $\Delta_{1}$ be the following set of translation definitions:

$$
\begin{gathered}
(\forall x) \operatorname{point}(x) \equiv \operatorname{Instant}(x) \\
(\forall x) \operatorname{line}(x) \equiv \operatorname{Interval}(x) \\
(\forall x, y) \operatorname{in}^{G}(x, y) \equiv(\operatorname{begins}(x, y) \vee \operatorname{ends}(x, y)) \\
(\forall x, y) \text { before }(x, y) \equiv \operatorname{lt}(x, y)
\end{gathered}
$$

Using Prover9 [11], we have shown that 6

$$
T_{\text {owltime_le }} \cup \Delta_{1} \models T_{\text {linear_order }} \cup T_{\text {loop_graphical }}
$$

Let $\Pi_{1}$ be the following set of translation definitions:

$$
(\forall x) \operatorname{Instant}(x) \equiv \operatorname{point}(x)
$$

\footnotetext{
${ }^{6}$ All proofs in this paper that have been generated by Prover9 can be found at http://stl.mie.utoronto.ca/colore/time/mappings/proofs /
} 


$$
\begin{gathered}
(\forall x) \text { Interval }(x) \equiv \operatorname{line}(x) \\
(\forall x, y) \operatorname{begins}(x, y) \equiv\left(\left(i n^{G}(x, y) \wedge\left((\forall z) i n^{G}(z, y) \supset l t(x, z)\right)\right.\right. \\
(\forall x, y) \text { ends }(x, y) \equiv\left(\left(i n^{G}(x, y) \wedge\left((\forall z) i n^{G}(z, y) \supset l t(z, x)\right)\right.\right. \\
(\forall x, y) \text { before }(x, y) \equiv l t(x, y)
\end{gathered}
$$

Using Prover9, we have shown that

$$
T_{\text {linear_order }} \cup T_{\text {loop_graphical }} \cup \Pi_{1} \models T_{\text {owltime_le }}
$$

Combining Theorem 6 and Theorem 4 gives us

Corollary 1. Towltime_leu is definably equivalent to $T_{\text {moment }}$.

Since all subtheories and extensions of $T_{\text {owltime_interval }}$ have the same nonlogical lexicon, we can use the same translation definitions as in the proof of Theorem 6 to prove the following theorem:

Theorem 7. Towltime_mleu is definably equivalent to

$$
T_{\text {linear_order }} \cup T_{\text {strong_graphical }}
$$

Combining Theorem 7 and Theorem 5 gives us

Corollary 2. $T_{\text {owltime_mleu }}$ is definably equivalent to $T_{\text {vila }}$.

\subsection{Semilinear Ordering on Instants}

If we now generalize these results to semilinear orderings on instants, then we need to consider a different class of structures for the reducibility theorem.

Definition 7. A semilinear betweenness relation $\mathbf{B}$ is a ternary relation that is definable in a semilinear ordering $\langle X,<\rangle$ by the formula

$$
(\forall x, y, z) B(x, y, z) \equiv(((x<y) \wedge(y<z)) \vee((z<y) \wedge(y<x)))
$$

The semilinear betweenness relation captures the notion of comparability among instants within the semilinear ordering. The key axiom of $T_{\text {owltime_interval }}$ is the one which states that for every two comparable points, there exists an interval of which they are the endpoints. This relationship between intervals and the ordering over instants is then captured by the following class of structures:

Definition 8. A closed semilinear graphical geometry is a structure $\mathbb{S}=\left\langle X, Y, \mathbf{B}, \mathbf{i n}^{\mathbf{G}}\right\rangle$ such that

1. $\mathbb{B}=\langle X, \mathbf{B}\rangle$ is a semilinear betweenness relation;

2. $\mathbb{I}=\left\langle X, Y, \mathbf{i n}^{\mathbf{G}}\right\rangle$ is a graphical incidence structure; 
3. triples of points in the extension of the betweenness relation $\mathbf{B}$ are incident with the same line in $Y$;

4. any triple of points that are incident with the same line in $Y$ are ordered by the betweenness relation $\mathbf{B}$.

The class of closed semilinear graphical geometries is axiomatized by $T_{\text {csgg }} 7$.

We did not need this class for extensions of $T_{\text {owltime_interval }}$ with linear orderings because all instants in a linear ordering are comparable. This is not the case with semilinear orderings - elements on different branches are not comparable.

We can use translation definitions similar to those from the proof of Theorem 6 to prove the reducibility theorem for $T_{\text {owltime_se: }}$

Theorem 8. The theory $T_{\text {owltime_se }}=T_{\text {owltime_interval_e }} \cup T_{\text {owltime_instant_s }}$ is definably equivalent to

$$
T_{\text {semilinear_order }} \cup T_{\text {csgg }}
$$

\subsection{Intervals without Begin and Ends}

The preceding sections have shown the reducibility of theories that are extensions of $T_{\text {owltime_e }}$ (which requires that all intervals have endpoints) rather than Towltime_interval (which allows intervals that do not have beginning or end instants). Nevertheless, we can use these results to provide a representation theorem for Towltime_interval.

Definition 9. Let $\mathbb{I}=\langle P, I$, in $\rangle$ be an incidence structure.

A line $\mathbf{l} \in I$ is solitary iff it is incident with a unique point.

A line $\mathrm{l} \in I$ is isolated iff it is not incident with any point.

Theorem 9. Any model $\mathcal{M}$ of $T_{\text {owltime_interval contains as substructures a unique }}$ model $\mathcal{N}$ of $T_{\text {owltime_e }}$ and a bipartite incidence structure $\mathbb{I}=\langle P, L \cup R \cup C$, in $\rangle$ such that

$$
\mathcal{M} \cong \mathcal{N} \cup \mathbb{I}
$$

where $L$ and $R$ are disjoint sets of solitary lines and $C$ is a set of isolated lines.

\subsection{Discussion}

If we consider the summary of results in Figure 2] we can see that $T_{\text {owltime_leu }}$ is the theory which is a common extension of both $T_{\text {owltime_interval }}$ and $T_{\text {vila }}$. In addition, $T_{\text {owltime_mleu }}$ is the common subtheory of $T_{\text {owltime_interval }}$ and $T_{\text {vila }}$; as such, it is intepretable by all existing time ontologies for points and intervals.

As a consequence of these results, we can see that $T_{\text {owltime_interval }}$ is not interpretable by all of the existing ontologies for timepoints and intervals; in other words, there are ontologies that are not definably equivalent to any consistent extension of $T_{\text {owltime_interval }}$. In particular, it is not interpretable by any time ontology that prevents the existence of moments [1], such as $T_{\text {endpoints }}$ from [9].

\footnotetext{
${ }^{7}$ http://stl.mie.utoronto.ca/colore/geometry/csgg.clif
} 


\section{Inside Intervals}

Moving on to the next module $T_{\text {owltime_inside }}$ of $T_{\text {owltime }}$, we encounter a new relation, inside, between instants and intervals. Before we introduce the classes of structures required to characterize the models of this module, we briefly discuss the problem of unintended models of $T_{\text {owltime_inside. }}$.

\subsection{A Critique of the Axioms}

Ordering on Instants Inside an Interval. Mace can be used to construct a model of $T_{\text {owltime_interval }}$ that satisfies the sentence

$\left(\exists t_{1}, t_{2}, i\right)$ inside $\left(t_{1}, i\right) \wedge$ inside $\left(t_{2}, i\right) \wedge \neg$ before $\left(t_{1}, t_{2}\right) \wedge \neg$ before $\left.\left(t_{2}, t_{1}\right) \vee\left(t_{1} \neq t_{2}\right)\right)$

that is, a model in which the Instants in an Interval are not linearly ordered, even though the axioms do entail the condition that the beginning and end instants themselves are linearly ordered.

We add the following axiom to $T_{\text {owltime }}$ to guarantee that all Instants in an Interval are linearly ordered:

$$
\begin{gathered}
\left(\forall t_{1}, t_{2}, i\right) \text { inside }\left(t_{1}, i\right) \wedge \operatorname{inside}\left(t_{2}, i\right) \\
\supset\left(\text { before }\left(t_{1}, t_{2}\right) \vee \text { before }\left(t_{2}, t_{1}\right) \vee\left(t_{1}=t_{2}\right)\right)
\end{gathered}
$$

Which Instants are Inside? Although Hobbs and Pan assert:

The concept of inside is not intended to include the beginnings and ends of intervals.

the axiomatization in $T_{\text {owltime_inside }}$ does not quite capture these intended models. While we can use Prover9 to show that the following sentence is entailed by $T_{\text {owltime }}$

$\left(\forall i, t_{1}, t_{2}\right) \operatorname{ProperInterval}(i) \wedge \operatorname{begins}\left(t_{1}, i\right) \wedge \operatorname{ends}\left(t_{2}, i\right) \supset \neg \operatorname{inside}\left(t_{1}, i\right) \wedge \neg \operatorname{inside}\left(t_{2}, i\right)$

Mace can be used to construct models of $T_{\text {owltime_inside }}$ that falsify each of the following sentences:

$$
\begin{gathered}
\left(\forall i, t_{1}\right) \operatorname{ProperInterval}(i) \wedge \operatorname{begins}\left(t_{1}, i\right) \supset \neg \text { inside }\left(t_{1}, i\right) \\
\left(\forall i, t_{1}\right) \operatorname{ProperInterval}(i) \wedge \operatorname{ends}\left(t_{1}, i\right) \supset \neg \text { inside }\left(t_{1}, i\right) \\
\left(\forall i, t_{1}\right) \operatorname{Interval}(i) \wedge \operatorname{begins}\left(t_{1}, i\right) \supset \neg \text { inside }\left(t_{1}, i\right) \\
\left(\forall i, t_{1}\right) \operatorname{Interval}(i) \wedge \operatorname{ends}\left(t_{1}, i\right) \supset \neg i n \operatorname{ide}\left(t_{1}, i\right)
\end{gathered}
$$

In other words, $T_{\text {owltime_inside }}$ is not strong enough to eliminate models in which only the beginnings or ends of intervals are included as instants inside the interval.

If we are to entail these sentences (which should follow from the original intuition), we need to extend $T_{\text {owltime }}$ with the following two sentences:

$$
\begin{gathered}
\left(\forall i, t_{1}, t_{2}\right) \text { inside }\left(t_{1}, i\right) \wedge \operatorname{begins}\left(t_{2}, i\right) \supset \text { before }\left(t_{2}, t_{1}\right) \\
\left(\forall i, t_{1}, t_{2}\right) \text { inside }\left(t_{1}, i\right) \wedge \operatorname{ends}\left(t_{2}, i\right) \supset \text { before }\left(t_{1}, t_{2}\right)
\end{gathered}
$$




\subsection{Semilinear Ordered Geometries}

When we used incidence structures to represent the models of $T_{\text {owltime_interval }}$, we only needed to worry about two instants that are incident with an interval, namely, the beginning and the end. For models of $T_{\text {owltime_inside, }}$ intervals may be incident with a larger set of instants that are linearly ordered. We therefore need to introduce a new class of structures.

Definition 10. A semilinear ordered geometry is a structure $\mathbb{L}=\left\langle X, Y, \mathbf{B}\right.$, in $\left.^{\mathbf{L}}\right\rangle$ such that

1. $\mathbb{B}=\langle X, \mathbf{B}\rangle$ is a semilinear betweenness relation;

2. $\mathbb{I}=\left\langle X, Y, \mathbf{i n}^{\mathbf{L}}\right\rangle$ is a weak bipartite incidence structure;

3. any triple of points that are incident with the same line in $Y$ are ordered by the betweenness relation $\mathbf{B}$.

The class of semilinear ordered geometries is axiomatized by $T_{\text {slog }} 8$.

\subsection{Reducibility Theorem for $T_{\text {owltime_inside }}$}

Let $T_{\text {owltime_inside }}^{*}$ be the axioms in $T_{\text {owltime_inside }} \cup T_{\text {owltime_se }}$ together with Axioms 1, 2, and 3 .

Theorem 10. $T_{\text {owltime_inside }}^{*}$ is definably equivalent to

$$
T_{\text {semilinear_order }} \cup T_{\text {csgg }} \cup T_{\text {slog }}
$$

Proof. Let $\Delta_{2}$ be the set of translation definitions in $\Delta_{1}$ together with:

$$
(\forall x, y) i n^{L}(x, y) \equiv \operatorname{inside}(x, y)
$$

$(\forall x, y, z) B(x, y, z) \equiv(($ before $(x, y) \wedge$ before $(y, z)) \vee($ before $(z, y) \wedge$ before $(y, z)))$

Using Prover9, we have shown that

$$
T_{\text {owltime_inside }}^{*} \cup \Delta_{2} \models T_{\text {semilinear_order }} \cup T_{\text {csgg }} \cup T_{\text {slog }}
$$

Let $\Pi_{2}$ be the set of translation definitions in $\Pi_{1}$ together with:

$$
(\forall x, y) \operatorname{inside}(x, y) \equiv i n^{L}(x, y)
$$

Using Prover9, we have shown that

$$
T_{\text {semilinear_order }} \cup T_{\text {csgg }} \cup T_{\text {slog }} \cup \Pi_{2} \models T_{\text {owltime_inside }}^{*}
$$

\footnotetext{
${ }^{8}$ http://stl.mie.utoronto.ca/colore/geometry/slog.clif
} 


\section{Eventualities}

Hobbs and Pan introduce the class of eventualities to "cover events, states, processes, propositions, states of affairs, and anything else that can be located with respect to time." In this section, we characterize the models of the two subtheories, $T_{\text {eventuality }}$ and $T_{\text {timespan }}$, of $T_{\text {owltime }}$ which axiomatize the intuitions for eventualities and their relationships to instants and intervals.

\subsection{Weak Planar Geometries}

Since $T_{\text {eventuality }}$ extends $T_{\text {owltime_interval }}$, a natural approach is to extend the geometries that are the underlying structures for the intended models.

Definition 11. A weak planar geometry is a tripartite incidence structure

$$
\mathbb{E}=\left\langle X, Y, Z, \mathbf{i n}^{\mathbf{E}}\right\rangle
$$

in which $N(\mathbf{q})$ is a linear ordered geometry for each $\mathbf{q} \in Z$.

Examples of weak planar geometries can be found in Figure 3 a) and 3 (b). In each example, the incidence relation between the eventuality $\mathbf{e}_{\mathbf{1}}$ and the intervals $\mathbf{i}_{\mathbf{j}}$ corresponds to the during relation; the incidence relation between $\mathbf{e}_{\mathbf{1}}$ and the instants $\mathbf{t}_{\mathbf{k}}$ corresponds to the atTime relation.

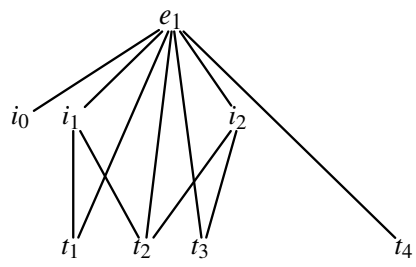

(a)

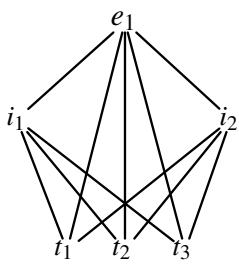

(b)

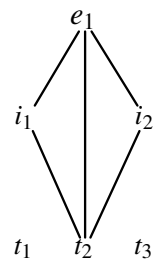

(c)

Fig. 3. Examples of weak planar geometries and diamond geometries

The class of weak planar geometries is axiomatized by $T_{\text {weak_planar }} 9$.

\subsection{Reducibility Theorem for $\boldsymbol{T}_{\text {eventuality }}$}

Let $T_{\text {eventuality }}^{*}$ be the axioms in $T_{\text {eventuality }} \cup T_{\text {inside }}^{*}$ together with

$$
\begin{gathered}
(\forall e) \text { Eventuality }(e) \supset \neg \text { TemporalEntity }(e) \\
(\forall e) \operatorname{atTime}(e) \supset \text { Eventuality }(e) \\
(\forall e) \text { during }(e) \supset \text { Eventuality }(e)
\end{gathered}
$$$$
(\forall t, e) \text { timeSpan }(t, e) \supset \text { Eventuality }(e) \wedge \text { TemporalEntity }(e)
$$

\footnotetext{
${ }^{9}$ http://stl.mie.utoronto.ca/colore/geometry/weak_planar.clif
} 
Theorem 11. $T_{\text {eventuality }}$ is definably equivalent to

$$
T_{\text {semilinear_order }} \cup T_{\text {csgg }} \cup T_{\text {slog }} \cup T_{\text {weak_planar }}
$$

Proof. Let $\Delta_{3}$ be the set of translation definitions in $\Delta_{2}$ together with:

$$
(\forall x, y) \operatorname{in}^{E}(x, y) \equiv(\operatorname{inside}(x, y) \vee \operatorname{during}(x, y) \vee \operatorname{atTime}(x, y))
$$

Using Prover9, we have shown that

$$
T_{\text {eventuality }} \cup \Delta_{3}=T_{\text {semilinear_order }} \cup T_{\text {csgg }} \cup T_{\text {slog }} \cup T_{\text {weak_planar }}
$$

Let $\Pi_{3}$ be the set of translation definitions in $\Pi_{2}$ together with:

$$
\begin{gathered}
(\forall x) \text { eventuality }(x) \equiv \operatorname{plane}(x) \\
(\forall x, y) \operatorname{during}(x, y) \equiv \operatorname{in}^{E}(x, y) \wedge \operatorname{plane}(x) \wedge \operatorname{line}(y) \\
(\forall x, y) \operatorname{atTime}(x, y) \equiv i n^{E}(x, y) \wedge \operatorname{plane}(x) \wedge \operatorname{point}(y)
\end{gathered}
$$

Using Prover9, we have shown that

$$
T_{\text {semilinear_order }} \cup T_{\text {csgg }} \cup T_{\text {slog }} \cup T_{\text {weak_planar }} \cup \Pi_{3} \models T_{\text {eventuality }}
$$

\subsection{Diamond Semilattices}

We can now proceed to the final class of structures that we will need to characterize models of $T_{\text {eventuality }}$ as part of the full representation theorem.

Definition 12. A diamond semilattice is a bounded semilattice in which all nonextremal elements are incomparable.

Definition 13. A diamond geometry is a tripartite incidence structure

$$
\mathbb{D}=\left\langle X, Y, Z, \mathbf{i n}^{\mathbf{D}}\right\rangle
$$

in which $N(\mathbf{q})$ is isomorphic to a diamond semilattice for each $\mathbf{q} \in Z$.

The class of diamond geometries is axiomatized by $T_{\text {diamond }}$.

Figure 3(c) is an example of a diamond geometry, which is isomorphic to the extension of the timespan relation; note that it is a substructure of the weak planar geometry in Figure 3(b). Figure 3 a) is an example of a weak planar geometry that does not contain a diamond geometry as a substructure; this corresponds to a model of $T_{\text {timespan }}$ in which the extension of the timespan relation is empty.

${ }^{10}$ http://stl.mie.utoronto.ca/colore/geometry/diamond.clif 


\subsection{Reducibility Theorem for $\boldsymbol{T}_{\text {timespan }}$}

Theorem 12. $T_{\text {timespan }}^{*}=T_{\text {timespan }} \cup T_{\text {eventuality }}^{*}$ is definably equivalent to $T_{\text {semilinear_order }} \cup T_{\text {csgg }} \cup T_{\text {slog }} \cup T_{\text {weak_planar }} \cup T_{\text {diamond }}$

Proof. Let $\Delta_{4}$ be the set of translation definitions in $\Delta_{3}$ together with:

$$
(\forall x, y) i \mathrm{in}^{D}(x, y) \equiv \operatorname{timeSpan}(x, y)
$$

Using Prover9, we have shown that

$$
T_{\text {timespan }}^{*} \cup \Delta_{4} \models T_{\text {semilinear_order }} \cup T_{\text {csgg }} \cup T_{\text {slog }} \cup T_{\text {weak_planar }} \cup T_{\text {diamond }}
$$

Let $\Pi_{4}$ be the set of translation definitions in $\Pi_{3}$ together with:

$$
\begin{gathered}
(\forall x, y) \operatorname{time} S p a n(x, y) \equiv\left(\operatorname { i n } ^ { D } ( x , y ) \wedge \operatorname { p l a n e } ( x ) \wedge \left((\exists z) \operatorname{line}(z) \wedge i n^{E}(z, x)\right.\right. \\
\left.\left.\wedge\left((\forall w) \operatorname{point}(w) \supset\left(\operatorname{in}^{E}(w, x) \equiv i n^{E}(w, z)\right)\right)\right)\right)
\end{gathered}
$$

Using Prover9, we have shown that

$$
T_{\text {semilinear_order }} \cup T_{\text {csgg }} \cup T_{\text {slog }} \cup T_{\text {weak_planar }} \cup T_{\text {diamond }} \cup \Pi_{4} \models T_{\text {timespan }}^{*}
$$

\section{Representation Theorem for $T_{\text {owltime }}$}

The reducibility of $T_{\text {timespan }}^{*}$ in Theorem 12 is the first step in the verification of the ontology The second step is to define the class of intended models:

Definition 14. $\mathfrak{M}^{\text {owltime }}$ is the following class of structures: $\mathcal{M} \in \mathfrak{M}^{\text {owltime }}$ iff

1. $\mathcal{M} \cong \mathcal{P} \cup \mathbb{G} \cup \mathbb{L} \cup \mathbb{E} \cup \mathbb{D}$, where

(a) $\mathcal{P}=\langle P,<\rangle$ is a linear ordering;

(b) $\mathbb{G}=\left\langle P, I\right.$, in $\left.^{\mathbf{G}}\right\rangle$ is a closed semilinear graphical geometry;

(c) $\mathbb{L}=\left\langle P, I, \mathbf{B}\right.$, in $\left.^{\mathbf{L}}\right\rangle$ is a semilinear ordered geometry;

(d) $\mathbb{E}=\left\langle P, I, E, \mathbf{i n}^{\mathbf{E}}\right\rangle$ is a weak planar geometry;

(e) $\mathbb{D}=\left\langle P, I, E, \mathbf{i n}^{\mathbf{D}}\right\rangle$ is a diamond geometry.

2. $\left\langle\mathbf{p}_{\mathbf{1}}, \mathbf{p}_{\mathbf{2}}\right\rangle \in$ before iff $\mathbf{p}_{\mathbf{1}}<\mathbf{p}_{\mathbf{2}}$;

3. $\langle\mathbf{p}, \mathbf{i}\rangle \in$ begins iff $\langle\mathbf{p}, \mathbf{i}\rangle \in$ in $^{\mathbf{G}}$ and for any $\mathbf{p}^{\prime} \in P$ such that $\left\langle\mathbf{p}^{\prime}, \mathbf{i}\right\rangle \in \mathbf{i n}^{\mathbf{G}}$, we have $\mathbf{p}<\mathbf{p}^{\prime}$;

4. $\langle\mathbf{p}, \mathbf{i}\rangle \in$ ends iff $\langle\mathbf{p}, \mathbf{i}\rangle \in$ in $^{\mathbf{G}}$ and for any $\mathbf{p}^{\prime} \in P$ such that $\left\langle\mathbf{p}^{\prime}, \mathbf{i}\right\rangle \in \mathbf{i n}^{\mathbf{G}}$, we have $\mathbf{p}^{\prime}<\mathbf{p}$;

5. $\langle\mathbf{p}, \mathbf{i}\rangle \in$ inside iff $\langle\mathbf{p}, \mathbf{i}\rangle \in$ in $^{\mathbf{L}}$;

6. $\langle\mathbf{e}, \mathbf{p}\rangle \in$ atTime iff $\mathbf{e} \in E, \mathbf{p} \in P$ and $\langle\mathbf{e}, \mathbf{p}\rangle \in \mathbf{i n}^{\mathbf{E}}$;

7. $\langle\mathbf{e}, \mathbf{i}\rangle \in$ during iff $\mathbf{e} \in E, \mathbf{i} \in I$ and $\langle\mathbf{e}, \mathbf{i}\rangle \in \mathbf{i n}^{\mathbf{E}}$;

8. $\langle\mathbf{t}, \mathbf{e}\rangle \in \operatorname{timeSpan}$ iff $\langle\mathbf{t}, \mathbf{e}\rangle \in \operatorname{in}^{\mathbf{D}}$ and $N(\mathbf{e}) \cong K_{1, m, n}$.

We can now state the Representation Theorem for $T_{\text {timespan }}^{*}$ : 
Theorem 13. $\mathcal{M} \in \mathfrak{M}^{\text {owltime }}$ iff $\mathcal{M} \in \operatorname{Mod}\left(T_{\text {timespan }}^{*}\right)$.

Proof. The theorem follows from Theorem 12 and Theorem 1 together with the fact that each of the substructures of $\mathcal{M} \in \mathfrak{M}^{\text {owltime }}$ corresponds to a theory in the reduction $-T_{\text {semilinear_order }}$ axiomatizes the class of semilinear orderings, $T_{\text {csgg }}$ axiomatizes the class of closed semilinear graphical geometries, $T_{\text {slog }}$ axiomatizes the class of semilinear ordered geometries, $T_{\text {weak_planar }}$ axiomatizes the class of weak planar geometries, and $T_{\text {diamond }}$ axiomatizes the class of diamond geometries.

\section{Summary}

The first-order time ontology for the Semantic Web proposed by Hobbs and Pan aims to be a core ontology for specifying temporal concepts for a wide variety of web applications and services. If it is to play this role effectively, we need a characterization of the models of the ontology and a guarantee that these models are equivalent to the intended models of the ontology's concepts. In this paper, we have provided a characterization of the models of $T_{\text {owltime }}$ up to isomorphism. This verification of $T_{\text {owltime }}$ has also led to modularization of the ontology and the identification of additional axioms to capture intuitions for intended models. We have also shown that two axioms of $T_{\text {owltime }}$ make it inconsistent with some existing time ontologies for points and intervals.

The next step is the proof of representation theorems for all of $T_{\text {owltine }}$, including the axioms for duration and dates, based on the duration and dates ontology in [8].

\section{References}

1. Allen, J., Hayes, P.: Moments and points in an interval-based temporal logic. Computational Intelligence 5, 225-238 (1989)

2. van Benthem, J.F.A.K.: The Logic of Time: A Model-Theoretic Investigation into the Varieties of Temporal Ontology and Temporal Discourse, 2nd edn. Springer, Heidelberg (1991)

3. Buekenhout, F.: Handbook of Incidence Geometry. Elsevier (1995)

4. Droste, M., Holland, W., MacPherson, H.: Automorphism groups of infinite semilinear orders. Proceedings of the London Mathematical Society 58, 454-478 (1989)

5. Enderton, H.: Mathematical Introduction to Logic. Academic Press (1972)

6. Gruninger, M., Hashemi, A., Ong, D.: Ontology Verification with Repositories. In: Formal Ontologies and Information Systems 2010, Toronto, Canada (2010)

7. Gruninger, M., Ong, D.: Verification of Time Ontologies with Points and Intervals. In: 18th International Symposium on Temporal Representation and Reasoning (2011)

8. Gruninger, M.: Ontologies for Dates and Duration. In: Twelfth International Conference on Principles of Knowledge Representation and Reasoning (2010)

9. Hayes, P.: A Catalog of Temporal Theories, Tech. Report UIUC-BI-AI-96-01, University of Illinois (1996)

10. Hobbs, J., Pan, F.: An Ontology of Time for the Semantic Web. ACM Transactions on Asian Language Information Processing 3, 66-85 (2004)

11. McCune, W.: Prover9 and Mace4 (2005-2010), http: / / www. cs. unm. edu/ mccune/Prover9

12. Pan, F., Hobbs, J.: Time in OWL-S. In: Proceedings of AAAI Spring Symposium on Semantic Web Services. Stanford University (2004)

13. Vila, L.: Formal Theories of Time and Temporal Incidence. In: Fisher, Gabbay, Vila, (eds.) Handbook of Temporal Reasoning in Artificial Intelligence. Elsevier (2005) 